\title{
Acoustic instrumentation for a bubble chamber towards dark matter searches
}

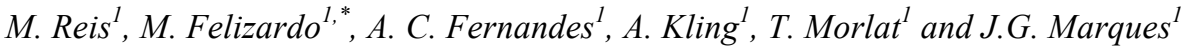 \\ ${ }^{1} \mathrm{C}^{2} \mathrm{TN}$, Instituto Superior Técnico, Universidade de Lisboa, E.N. 10 (km 139.7), 2695-066 Bobadela, \\ LRS, Portugal
}

\begin{abstract}
Dark matter is one of the top unsolved mysteries in physics. Its existence is well-established although its nature remains unknown. Despite the progress made in the direct search effort, reflecting over 10 orders of magnitude in sensitivity since 1984 , no true candidates to explain this phenomenon have appeared in searches covering a range from $\sim 10 \mathrm{GeV}$ to 1 $\mathrm{TeV}$. This article reports on the development of a $1 \mathrm{~kg}$ freon bubble chamber prototype, including the chamber recompression system design and testing, initial acoustic detection of bubble formation, and initial neutron and alpha detector response studies. The prototype constructed was a transparent acrylic containment vessel, capable of withstanding recompression cycles to a pressure of 16 bar. The acoustic signal accompanying bubble formation was investigated using three different sensors: a low frequency microphone (Panasonic) with a flat response over $0.020-16 \mathrm{kHz}$, an ultrasound externallypolarized condenser microphone (AviSoft) with a flat response over 10-150 $\mathrm{kHz}$, and an hydrophone (Reson) with a flat response over 5-170 kHz. Acoustic signatures of several induced events were successfully registered. The data acquisition digitizer used, to meet the range of the three microphones, was the NI PCI-6251 16-Bit, with at least 1.25 MSps for 1Channel.
\end{abstract}

\section{Introduction}

In 2012, the SIMPLE Collaboration [1] imposed the most stringent SpinDependent constraint on the dark matter exclusion plot, contributing to reduce the possible window of existence of a WIMP (weakly interacting massive particle) to explain the dark matter phenomenon. SIMPLE obtained this result while working with superheated droplet detectors (SDDs) although, to remain competitive with other experiments in the field, it needed to increase the total active mass. The encountered solution was to substitute the SDDs in the search for dark matter, with a bubble chamber, increasing the active mass in more than $2000 \%$. This article describes precisely the complete deployment, i.e., construction, instrumentation, and analysis of acoustic results from particle calibrations, of a bubble chamber detector for dark matter searches. 


\section{Dark Matter}

According to the latest data provided by ESA's Planck satellite [2], dedicated to the study of the early Universe and its subsequent evolution, the baryonic matter constitutes only $4.9 \%$ of the total amount of matter and energy in the Universe. The largest contribution, $68.3 \%$ is assured by dark energy, which is thought to be the cause of the accelerated expansion of the universe. In addition, the remaining percentage, $26.8 \%$, is attributed to a type of matter that does not interact with light and has not yet been seen experimentally - dark matter (DM).

Many efforts have been made to detect an experimental signature of dark matter, some through direct detection experiments, via scattering of a DM particle on a target nuclei, others through indirect means, by searching for signals from annihilation or decay products of such a particle. So far, none of these experiments have been able to detect the desired experimental signature being only able to impose constraints in the mass spectrum. The processes of DM particle annihilation or decay originates neutrinos, gamma rays, positrons, electrons, among other particles. The strongest limits on high energy neutrinos coming from the Sun are placed by IceCube and Super-Kamiokande. For highly energetic gammarays $(\mathrm{TeV})$ upper limits have been derived by the indirect detection experiments MAGIC, HESS and VERITAS telescopes.

Direct detection experiments study the nuclear recoil produced by dark matter particle scattering. The recoil of the nucleus is converted into, for example, electrons, photons, and phonons, which can be detected by ionization, scintillation and thermal detectors, respectively. Among those with highest sensitivities are cryogenic experiments operated at sub-Kelvin temperatures; detectors based on liquid argon or xenon; and superheated liquid detectors. The low mass region of the spectrum is accessible by detectors with very low energy threshold and/or lighter target nuclei, while the higher mass region is probed by experiments with very low background rates and high target masses. For low WIMP masses (below $10 \mathrm{GeV}$ ), the tightest constraints are derived from CRESST, EDELWEISS and SuperCDMS. The most constraining upper limits for WIMP masses come from the LUX experiment using a liquid xenon time-projection chamber.

The current experimental upper limits for spin-dependent WIMP-proton and spinindependent WIMP-nucleon cross-sections, as a function of the WIMP mass, are summarized in Fig.1.
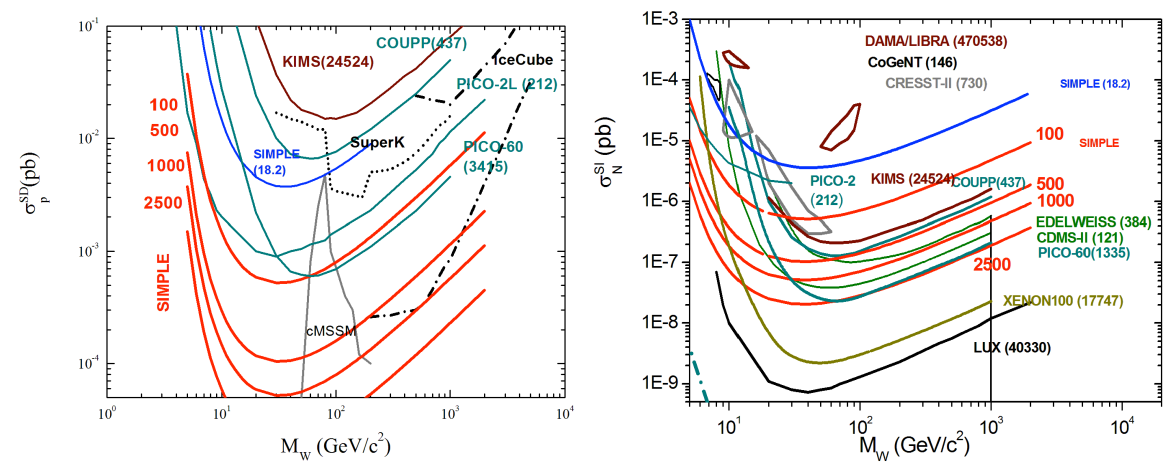

Fig. 1. Spin-dependent limits on the WIMP-proton scattering cross-section (left); Spin-independent WIMP-nucleon scattering (right), both as a function of the WIMP mass. Figure from [3]. 
The spin-dependent (SD) interactions result from the coupling of a WIMP's spin to the spin content of a nucleon. The cross-section for spin-dependent interactions is proportional to $\mathrm{J}(\mathrm{J}+1)$, $\mathrm{J}$ being the total nuclear spin, rather than to the number of nucleons, so, little is gained by using heavier target nuclei. On the contrary, for spin-independent (SI) scattering the cross-section does not depend on spin and increases dramatically with the mass of the target nuclei.

The most competitive experiments in the spin-dependent channel are PICO [4] and SIMPLE [5]. These are both superheated liquid detector experiments. PICO uses a bubble chamber filled with $\mathrm{C}_{3} \mathrm{~F}_{8}$ and SIMPLE uses superheated droplet detectors (SDDs) with Freon $\mathrm{C}_{2} \mathrm{ClF}_{5}$ as their active mass. Taking into account Fig.1, one can see the SIMPLE projection curves, represented in red, for a "zero background" 100, 500, 1000, 2500 kilogram.day (kgd) exposure measurements.

These curves give sensitivities of order $10^{-4} \mathrm{pb}$ for SD and $10^{-7} \mathrm{pb}$ for SI. The projections represent the ambition of the new phase of SIMPLE, in which the SDDs are replaced by bubble chamber (BC) detectors. This will allow an increase in active mass and a decrease in data acquisition time.

\section{Bubble Chamber Deployment}

As a container for this DM detector, a 1.7L PET (polyethylene terephthalate) recipient, resistant up to 16 bar of pressure, sold commercially for water filtration systems, was used. These types of containers are the ones usually used by the SIMPLE Collaboration, the only difference is that the previous ones could only go up to 10 bar of pressure and the one employed in this work goes up to 16 bar. This rise in maximum pressure is absolutely crucial when it comes to a BC detector with the amount of active mass used in this work ( $\sim 18 \%$ of the detector's volume). We used, for the chamber's active mass, $\sim 300 \mathrm{~g}$ of two different types of superheated liquids, $\mathrm{C}_{2} \mathrm{ClF}_{5}$ and $\mathrm{C}_{3} \mathrm{~F}_{8}$. The recompression system needs to be powerful and fast enough to operate this detector without enormously increasing its dead time. The gel used to cover this chamber walls, was the same as the one employed by the SIMPLE Collaboration in the fabrication of the superheated droplet detectors [6].

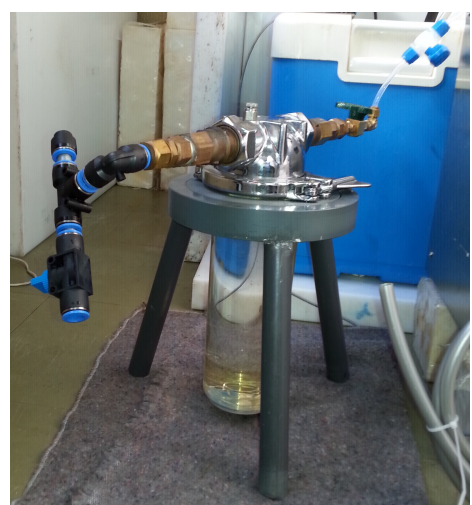

Fig. 2. Chamber on a tripod, with all the connections of the cap assembled. The yellow tonality observed, especially in lower part of the chamber's body, is due to the gel covering the chamber walls and bottom. 
The automaton that controls the bubble chamber detector, responsible for its recompression, is essentially operated with two glycerin pistons. Both these Festo components compress or release pressure of the chamber, as the software routines demand, and are compressed with pressurized $\mathrm{N}^{2}$ gas. Each piston is connected to a different pressure line, the blue line is used for low pressure values (Fig.3) and the green one for the higher pressure values (assembled symmetrically on the back of the blue line system of Fig.3). To connect all the components, several high pressure Festo tubing, connectors, and fittings were used. Figure 3 presents a real picture of the automaton that controls the chamber operation and it is possible to see all these connectors and fittings in detail. To help compensate the glycerin flow, the system has incorporated a glycerin reservoir, which also has the capability to purge unwanted $\mathrm{N}^{2}$ from the system. The interface with the automaton is made using a soft programming software built with Crouzet Logic Software M3. The two necessary routines to operate the bubble chamber were created using this software. After many changes and different versions, the routine OPEN Valves allows the user to control the opening and closure of all the electro valves of the automaton, and the routine Chamber Operation is the one used for the chamber's normal operation.

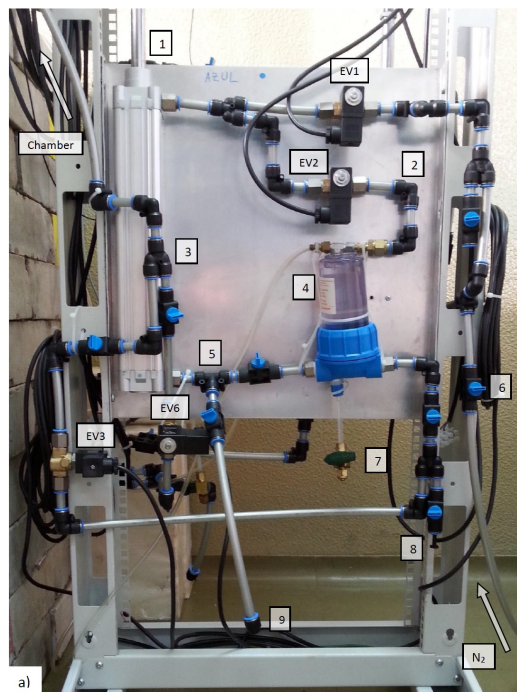

Fig. 3. Real picture of the automaton's blue line, where the piston goes from 2 to 8 bar. The $\mathrm{N}^{2}$ gas used to compress the piston gets in the system through the tube on the right, straight up to EV1. This $\mathrm{EV}$ is the one controlling the amount of $\mathrm{N}^{2}$ used to compress the blue piston and EV2 releases the $\mathrm{N}^{2}$ when the chamber is de-compressing. The tube on the upper left corner is the one going straight to the chamber to apply the pressure changes made by both pistons. EV3 applies the pressure changes made by the blue piston and EV6 applies the ones made by the green piston. Caption: 1- blue piston, 2- Lconnector (elbow), 3- Y-connector, 4- glycerine reservoir, 5- T-connector, 6- manual valve, 7- purge, 8- blanking plug, 9-cap.

SIMPLE pioneered the acoustic recording of the event signals [7]. The events are recorded via two channels: acoustically, using two different types of microphones and one hydrophone, and visually using a GoPro camera. The shock wave produced by the bubble nucleation and expansion can be recorded by an acoustic sensor and then converted into an electrical pulse. This pulse tends to be a damped sinusoidal with a typical duration of several milliseconds. The COUPP experiment demonstrated [8] that the sound of the acoustic event associated with a gas bubble nucleation in a bubble chamber shows a broad 
emission up to a frequency of $250 \mathrm{kHz}$. To record the bubble nucleation events, the following acoustic devices were used: an ultrasound, externally-polarized condenser microphone (CM16/ CMPA40-5V from Avisoft- Bioacoustics) with a flat response over the $10-150 \mathrm{kHz}$ frequency range, to record the shock wave associated with protobubble formation; a low frequency microphone, from Panasonic (omnidirectional) with a frequency response over the range of $0.020-16 \mathrm{kHz}(3 \mathrm{~dB})$, with a signal-to-noise ratio (SNR) of $58 \mathrm{~dB}$ and a sensitivity of $7.9 \mathrm{mV} / \mathrm{Pa}$ at $1 \mathrm{kHz}$; and an hydrophone (Reson) with a flat response over 5-170 kHz. To visually record the events, a GoPro Hero 5 black edition camera, waterproof resistant up to 30 feet of depth, with a system of focus free and a maximum video resolution of 3840 x 2160 with 30 frames per second was used. To make the bridge between the electronic devices and the computer acquiring the data, a digitizer NI-DAQ was used. In order to meet the range of the three microphones used, the DAQ board employed was the NI PCI-6251 16- Bit, with at least 1.25 MSps for 1-Channel.

\section{Signal \& Result Analysis}

After studying the background noise of the laboratory, tests to the acoustic recording devices were performed, first by using a function generator and afterwards by simulating nucleations creating air bubbles inside water with a straw. The result of these initial tests allowed us to take conclusions about the quality of the signal acquired by the three acoustic recording devices, and analyze possible time delays, shifts or malfunctionings. Once the functioning of the three acoustic devices used was understood, a pressure stability test was performed to the chamber to guarantee that it was stable enough to only trigger events at the operation pressure. The test was successful and so one proceeded to the acquisition of nucleation signals. Figure 4 shows a typical 10 minute acquisition. It is visible that every time the pistons are requested to do the work in the chamber, the acoustic devices record a noise. This noise is precisely associated with the input/release of $\mathrm{N}^{2}$ gas into the system, used to operate the pistons. The recompression, done at 16 bar, takes approximately $30 \mathrm{sec}$.

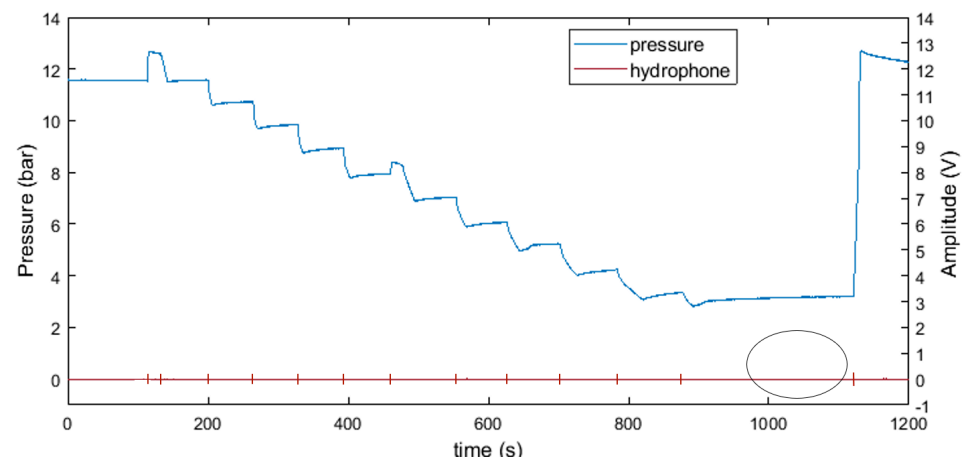

Fig. 4. Typical Acquisition.

In order to find the event, one has to look before the pressure rise (recompression) and before the piston noise associated with it (region represented with a circle in Fig.4). After manually finding a symmetric peak on the acoustic recording devices spectra, one has to isolate it and recur to Matlab to find its beginning and end in time. With the peak isolated in time, one applies a Fast Fourier Transform (FFT) to determine its frequency. The next step is to build a frequency filter around this value and apply it to the whole signal, obtaining a clearer visualization of the peak. An example is shown in Fig.5. 


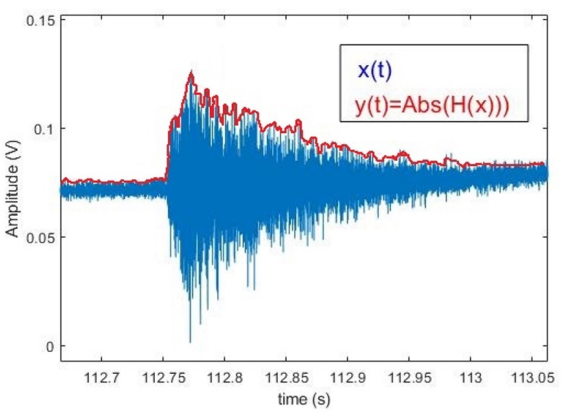

Fig. 5. Pulse signal of a typical event. In red, is represented the amplitude envelope.

To extract information about the characteristics of the event, one uses a simple pulse shape identification outline in which each pulse is first amplitude demodulated and then, its decay constant $(\tau)$ can be determined through an exponential fit. The amplitude demodulation is reached by performing the modulus of the Hilbert transform of the pulse waveform, $\mathrm{y}(\mathrm{t})=|\mathrm{H}\{\mathrm{x}(\mathrm{t})\}|$ giving the amplitude envelope. Moreover, $\tau$ can be obtained, once found the maximum and minimum of the pulse shape, defining the time window of the pulse. The decaying part of the amplitude envelope is then fit to an exponential function, according to,

$$
h(t)=\mathrm{Ae}^{-\mathrm{t} / \tau}
$$

Figure 6 shows both the amplitude envelope and the exponential fit to Eq. (1) which allows to determine $\tau$.

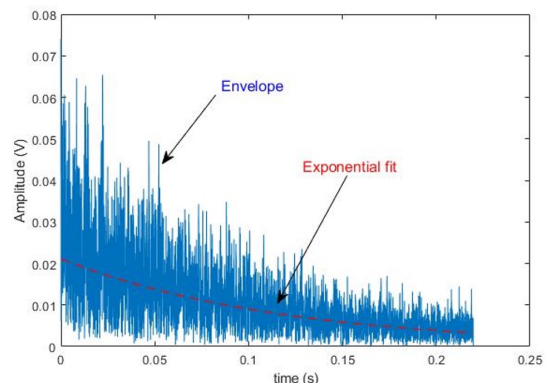

Fig. 6. Best fit of the exponential in Eq. (1) to the amplitude envelope of the pulse shown in Fig.5.

After isolating a single nucleation event, as the one shown in Fig.5, and making a best fit of the amplitude envelope, the main Power Spectral Density (PSD) response is found at $\sim 4.4 \mathrm{kHz}$ with a $\tau$ of $\sim 117.5 \mathrm{~ms}$. This is the typical frequency spectrum of bubble nucleation signals.

\section{Neutron \& Alpha Calibrations}

With the used conditions of pressure and temperature $\left(2.5\right.$ bar at $9^{\circ} \mathrm{C}$ for $\mathrm{C}_{2} \mathrm{ClF}_{5}$ and $12^{\circ} \mathrm{C}$ for $\mathrm{C}_{3} \mathrm{~F}_{8}$ ), the chamber is insensible to most atmospheric background radiation, remaining sensible to alpha particles and neutrons. The alpha particles will constitute the background events and the neutrons are thought to be particles with an experimental signature similar to the WIMPs, thus, it is important to calibrate with these sources. Regarding a study of the background radiation of the laboratory and the materials used, which has been done for the GESA facility in LSBB $[9,10]$, one can predict the number of expected events and analyze if there was an excess, which could be related to a passage of a WIMP. 
The SIMPLE Collaboration has done an extensive work in discriminating particle events with SDDs [11] and concluded that it is possible to distinguish between an alpha particle and a neutron event. They have different experimental signatures. Namely, the alpha particle produces more proto-bubbles therefore creates more energy inside a bubble nucleation, meaning the signal will have a larger amplitude, when comparing to a neutron induced event. In this paper we also present such a discrimination study, between alpha and neutron particles, made for the bubble chamber. The tests using the weak $\mathrm{AmBe}(0.1 \mathrm{mCi})$ neutron source were performed carefully placing the source inside the laboratory and shielding it with polyethylene plates. It stood at a distance of approximately 50 centimetres from the chamber. The overall frequencies and time constants, for the neutron induced events, were found to be approximately $4-15 \mathrm{kHz}$ and $75-150 \mathrm{~ms}$, respectively. As for the amplitudes of the acoustic pulses, these vary between $15-140 \mathrm{mV}$. These results are highly dependent on the size of the bubble that is formed upon radiation deposition. From what was observed visually, and recorded via the GoPro camera, smaller bubbles have lower amplitude signals and time constants, but higher frequencies. Larger bubbles give us the opposite result. After doing the neutron calibrations, what followed was an alpha calibration with a liquid source, introduced in the chamber via the superheated liquid injection line. The frequencies of the alpha induced events oscillate between 5 and $17 \mathrm{kHz}$ and have time constants that vary between $75-165 \mathrm{~ms}$. As for the amplitudes, 70-610 mV, they are in the majority of cases $\sim 40 \%$ higher - depending on the acoustic sensor - than the neutron induced events, thus having added power. The variation behaviour for the amplitudes, time constants and frequencies for the alpha induced events is the same as the one found, and mentioned above, for the neutron induced events, suggesting a strong relation between these parameters and the size of the bubble that is formed. For comparison purposes, in what follows, Fig. 7 shows an histogram considering the normalized number of events as a function of power.

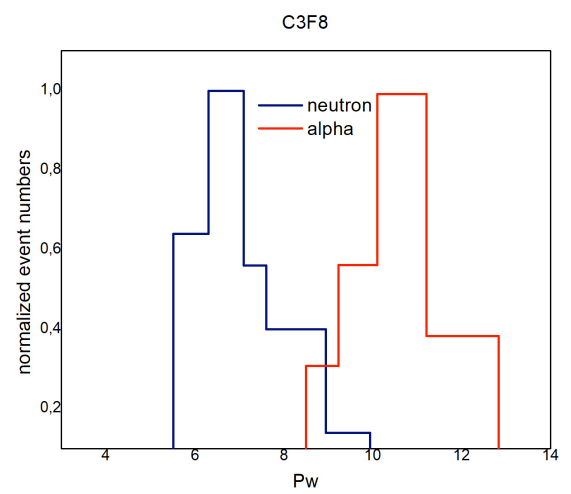

Fig. 7. Histogram presenting the calibrations made using alpha-particle and neutron sources.

The power is directly related with the pulse amplitudes, A, of the events (in $\mathrm{mV}$ ) and can be calculated according to,

$$
\mathrm{P} w=\ln \left(\mathrm{A}^{2}\right)
$$

These results were obtained at $(2.75-3.00)$ bar and $(12-16)^{\circ} \mathrm{C}$. As one can see from Fig.7, there is not yet a full discrimination between the events induced by alpha particles and the ones induced by neutrons. A possible reason for this to be happening, à priori, is the fact that the experiments were not performed under the same conditions of temperature. These series of measurements using calibrated alpha and neutron irradiations should continue to confirm the ability to fully distinguish both event types acoustically. 


\section{Conclusions}

The work presented in this paper shows the performance of a bubble chamber that allows to be operated at higher pressures (16 bar) than the ones used previously in the SIMPLE laboratory (10 bar). This allows the chamber to be recompressed faster and more efficiently, reducing the detector dead time by a factor of 6 (compared to the previous 10 bar). An automaton recompression system was created to operate the chamber. This involved the creation of a parallel system of pistons to compress the chamber to 16 bar. To operate this automaton, two routines were implemented. To instrument the bubble chamber, two new acquisition channels were introduced, assured by two different recording devices. The visual acquisition channel is assured by a water resistant camera that is able to accompany the detector underwater, into the bath, and record the events live. The acoustic channel, was enriched by the presence of an hydrophone that is also able to accompany the detector inside water, or even be placed inside it, to record the events directly from the inside. The acquired signal from the hydrophone was compared with the signals acquired from two extended microphones placed outside the chamber. Throughout the entire set of experiments done with the three acoustic sensors, the one that captured the majority (95\%) of the events was the hydrophone, since it is the one that is capable of being in close contact with the bubble chamber inside its temperature bath. As for the other two they were placed on the top lid of the $\mathrm{BC}$ and failed the ability of recording the low amplitude (smaller nucleations) events. Hints of a possible discrimination between alpha particles and neutrons has been shown. Although additional investigations have to be performed, the path for this optimized detector is clearly the path for dark matter searches. A discrimination towards the spatial localization [12] of the bubble inside the chamber is also to be evaluated in the near future.

This work is supported by $\mathrm{C}^{2} \mathrm{TN} / \mathrm{IST}$ and by grants PTDC/FIS/115733/2009, PTDC/FIS/121130/2010, PTDC/EEI-ELC/2468/2014 and IF/00628/2012/CP0171/CT0008. $\mathrm{C}^{2} \mathrm{TN} / \mathrm{IST}$ authors gratefully acknowledge the FCT support through the UID/Multi/04349/2013 project. The activity of M. Felizardo is supported by FCT grant SFRH/BPD/94028/2013.

\section{References}

1. M. Felizardo, et al. Phys. Rev. Lett. 108, 201302 (2012)

2. R. Adam, et al. Astronomy \& Astrophysics, 594 (2015)

3. M. Felizardo, et al. E3S W. C. 12, 03002, 3-8, (2016)

4. C. Amole et al. Phys. Rev. Lett. 118, 251301 (2017)

5. M. Felizardo, et al. Phys. Rev. D 89, 072013 (2014)

6. M. Felizardo et. al. Nucl. Instr. \& Meth. Physics Research A 614, 278-286, (2010)

7. M. Felizardo, R. C. Martins, A. R. Ramos, et al. Nucl. Instr. \& Meth. Physics Research A 585, 61-68, (2008)

8. E. Behnke et. al. Phys. Rev. D, 86, 052001, (2012)

9. A. C. Fernandes et. al. Astroparticle Physics, 76, 48-60, (2016)

10. A. C. Fernandes et. al. Nucl. Instr. \& Meth. Physics Research A 623, 960-967, (2010)

11. M. Felizardo, et al. Nucl. Instr. \& Meth. Physics Research A 863, 62-73, (2017)

12. M. Felizardo, R. C. Martins, T. A Girard, et al. Nucl. Instr. \& Meth. Physics Research A 599, 93-99, (2009) 